\title{
AN SFDI OBSERVER-BASED SCHEME FOR A GENERAL AVIATION AIRCRAFT
}

\author{
MARCO ARIOLA $^{a, *}$, MASSIMILIANO MATTEI $^{b}$, IMMACOLATA NOTARO $^{b}$, \\ FEDERICO CORRARO $^{c}$, AdOLFO SOLLAZZO $^{c}$ \\ ${ }^{a}$ Department of Engineering \\ University of Naples Parthenope, Isola C4, 80143 Naples, Italy \\ e-mail: ariola@uniparthenope.it \\ ${ }^{b}$ Department of Industrial and Information Engineering (DIII) \\ Second University of Naples, Real Casa dell'Annunziata Via Roma, 29, Aversa (CE), 81031, Italy \\ e-mail: \{massimiliano.mattei, immacolata.notaro\}@unina2.it \\ ${ }^{c}$ Guidance, Navigation and Control Laboratory \\ Italian Aerospace Research Center (CIRA), Capua (CE), Italy \\ e-mail: \{f.corraro,a.sollazzo\}@cira.it
}

\begin{abstract}
The problem of detecting and isolating sensor faults (sensor fault detection and isolation-SFDI) on a general aviation aircraft, in the presence of external disturbances, is considered. The proposed approach consists of an extended Kalman observer applied to an augmented aircraft plant, where some integrators are added to the output variables subject to faults. The output of the integrators should be ideally zero in the absence of model uncertainties, external disturbances and sensor faults. A threshold-based decision making system is adopted where the residuals are weighted with gains coming from the solution to an optimization problem. The proposed nonlinear observer was tested both numerically on a large database of simulations in the presence of disturbances and model uncertainties and on input-output data recorded during real flights. In this case, the possibility of successfully applying the proposed technique to detect and isolate faults on inertial and air data sensors, modelled as step or ramp signals artificially added to the real measurements, is shown.
\end{abstract}

Keywords: model-based FDI, sensor FDI, extended Kalman filtering, analytical redundancy, flight control.

\section{Introduction}

In the aircraft industry very stringent safety requirements are requested in system design. For this reason, solutions are typically based on physical redundancy, also named hardware redundancy. For example, the Boeing 777 flight system is a fly-by-wire system based on triple redundancy for all hardware resources: the computing system, the airplane electrical power, the hydraulic power and the communication path (Yeh, 1996). The inertial and air data sensors have a similar level of redundancy. Of course, this approach has the drawback of increasing the system weight, size and complexity. Moreover, the production costs are extremely high. Approaches alternative to hardware redundancy are being developed; the most promising is the so-called analytical redundancy, where

\footnotetext{
* Corresponding author
}

model-based and data-driven techniques are used to obtain the redundant measurements, hence reducing the reliance on physical redundancy. The use of analytical fault detection and isolation (FDI) algorithms would represent a significant change in the way this problem is tackled by the aerospace industry. One critical aspect which is presently slowing down this process is the need to certify the airworthiness of safety-critical systems.

In order to achieve an analytical sensor redundancy it is necessary to have at one's disposal one or more signal reconstruction modules, that is, one or more algorithms able to estimate a given variable from the knowledge of the inputs and the outputs of the system under consideration. This paper focuses on sensor failures on aircraft, which are not uncommon and may be extremely critical for safety. Several techniques are available in the literature to design state estimators for 
the solution of FDI problems (Chen and Patton, 1999; Basseville and Nikiforov, 1993; Isermann, 1984; Gertler, 1998; Gustafsson, 2000), among which the best assessed are those based on parity spaces (Gertler, 1997; Patton and Chen, 1994; Simani et al., 2003), observer-based methods (Clark et al., 1975; Wünnenberg and Frank, 1987), and approaches based on Kalman filters as well as $H_{2}$ or $H_{\infty}$ theories (Mehra and Peschon, 1971). These approaches have been extended also to nonlinear observers (see, e.g., Mattei et al., 2005).

Within the observer-based FDI approach, several schemes have been proposed in the literature:

- Dedicated observer scheme (DOS) (Clark et al., 1975): here, each observer is driven by a different output. In the event of a fault, the corresponding observer will produce inaccurate estimates, and therefore fault detection and isolation are made possible.

- Generalized observer scheme (GOS) (Wünnenberg and Frank, 1987): this scheme, as the DOS, makes use of a bank of observers. In this case, however, each observer is driven by all outputs except one. In this way, when an output is faulty, the estimates of all the observers except one are inaccurate.

- Simplified detection scheme (Clark, 1978): this scheme is a particular case of the DOS, and makes use of only one observer driven by only one measurement. Therefore, if any other measurement is faulty, the corresponding residual is non-zero. Of course, if the sensor used by the observer is faulty, then all the residuals are non-zero and no information can be gathered.

All these schemes can be used with Luenberger observers (Luenberger, 1971), Kalman filters (Kalman, 1960), unknown input observers (UIOs) (Watanabe and Himmelblau, 1982), or any other kind of state or output observer.

In this paper we propose the use of an extended Kalman filter approach for the detection and isolation of sensor faults modeled as step signals (abrupt faults) or ramp signals (incipient faults) added to the sensor measurements. The aircraft model is augmented introducing a bank of integrators, one on each variable subject to a fault. This allows us to use only one dynamic observer which is driven by all the outputs at the same time. In the ideal case of the absence of model uncertainties, external disturbances and sensor faults, the additional integrators have null steady-state states. When a fault on one variable occurs, the corresponding integrator starts charging; in this way, as shown in the paper, we are able not only to detect a fault, but also to isolate it. The scheme is applied to both simulated and experimental flight data of the general aviation aircraft Tecnam P92 (see Fig. 1).

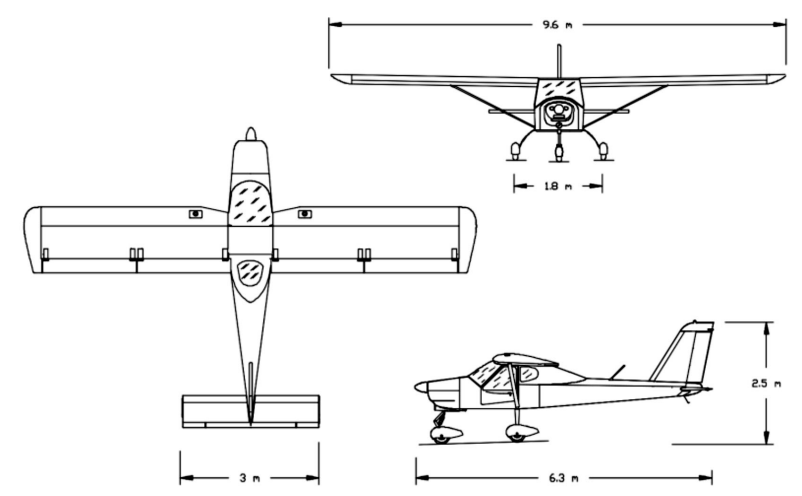

Fig. 1. Tecnam P92 aircraft.

The paper is organized as follows. In Section 2 the aircraft model is presented. In Section 3 the proposed observer scheme is discussed. Section 4 contains some simulation results obtained in the presence of model uncertainties, sensor noise and wind gusts. In Section 5] the proposed observer is applied to some flight experimental data and the faults are isolated using a logical decision making process based on thresholds obtained as solution of an optimization problem.

\section{Aircraft model}

The mathematical model of the GA aircraft under consideration is a classical six-degrees-of-freedom aircraft model.

In order to write the system equations in a compact form, we introduce the state vector

$\mathbf{x}=\left(\begin{array}{llllllllllll}u & v & w & p & q & r & \phi & \theta & \psi & x & y & z\end{array}\right)^{T}$,

the input vector

$$
\mathbf{u}=\left(\begin{array}{lllll}
\delta_{e} & \delta_{a} & \delta_{r} & \delta_{f} & \delta_{T}
\end{array}\right)^{T},
$$

and the wind disturbance vector

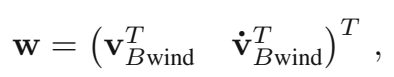

where

$$
\mathbf{v}_{B \text { wind }}=\left(u_{B \text { wind }} \quad v_{B \text { wind }} \quad w_{B \text { wind }}\right)^{T} .
$$

The symbols used are listed in Table 1.

The most significant external disturbances are sudden wind gusts, atmospheric turbulence and sensor noise. In the presence of atmospheric disturbances we have

$$
\begin{aligned}
V & =\left\|\mathbf{v}_{B}-\mathbf{v}_{B \text { wind }}\right\|, \\
\alpha & =\arctan \left[\left(w-w_{B \text { wind }}\right) /\left(u-u_{B \text { wind }}\right)\right], \\
\beta & =\arcsin \left[\left(v-v_{B \text { wind }}\right) / V\right],
\end{aligned}
$$


Table 1. List of symbols.

\begin{tabular}{|l||l|}
\hline$l_{c}$ & Reference length for aerodynamic moments \\
\hline$S$ & Wing reference area \\
\hline$V$ & True air speed \\
\hline$m$ & Aircraft mass \\
\hline $\mathbf{v}_{B}$ & Velocity vector in body axes: $\mathbf{v}_{B}=\left(\begin{array}{ll}u & w\end{array}\right)^{T}$ \\
\hline$\omega$ & Angular velocity in body axes: $\omega=(p q r)^{T}$ \\
\hline $\boldsymbol{\Theta}$ & Euler angles: $\boldsymbol{\Theta}=(\phi \theta \psi)^{T}$ \\
\hline$\zeta$ & Position in inertial frame of reference: $\zeta=(x y z)^{T}$ \\
\hline $\mathbf{v}_{B \text { wind }}$ & Wind velocity vector in body axes \\
\hline $\mathbf{a}_{B}$ & Acceleration in body axes: $\mathbf{a}_{B}=\left(a_{x} a_{y} a_{z}\right)^{T}$ \\
\hline $\mathbf{F}$ & Forces vector \\
\hline $\mathbf{J}$ & Inertia matrix \\
\hline $\mathbf{T}$ & Moments vector \\
\hline $\mathbf{R}_{B E}$ & Transformation matrices from earth to body axes \\
\hline$\alpha$ & Angle of attack \\
\hline$\beta$ & Sideslip angle \\
\hline$\rho$ & Air density \\
\hline$\delta_{e}, \delta_{a}, \delta_{r}$ & Elevator, Ailerons, Rudder deflection (primary surfaces) \\
\hline$\delta_{f}$ & Flap deflection \\
\hline$\delta_{T}$ & Throttle command \\
\hline
\end{tabular}

with $\|\cdot\|$ denoting the Euclidean norm. The sensor noise was modelled as Gaussian white noise with variance determined based on registered flight data.

Using the symbols introduced above, we can write the equations of motion in body axes in the form (see Stevens and Lewis, 2003)

$$
\begin{aligned}
& \left(\begin{array}{c}
\dot{x}_{1} \\
\dot{x}_{2} \\
\dot{x}_{3}
\end{array}\right)=\frac{1}{m}\left(-\left(\begin{array}{c}
x_{4} \\
x_{5} \\
x_{6}
\end{array}\right) \times\left(\begin{array}{c}
x_{1} \\
x_{2} \\
x_{3}
\end{array}\right)+\mathbf{F}(\mathbf{x}, \mathbf{u}, \mathbf{w})\right), \\
& \left(\begin{array}{l}
\dot{x}_{4} \\
\dot{x}_{5} \\
\dot{x}_{6}
\end{array}\right)=\mathbf{J}^{-1}\left(-\left(\begin{array}{l}
x_{4} \\
x_{5} \\
x_{6}
\end{array}\right) \times \mathbf{J}\left(\begin{array}{l}
x_{4} \\
x_{5} \\
x_{6}
\end{array}\right)+\mathbf{T}(\mathbf{x}, \mathbf{u}, \mathbf{w})\right) \text {, } \\
& \left(\begin{array}{c}
\dot{x}_{7} \\
\dot{x}_{8} \\
\dot{x}_{9}
\end{array}\right)=\left(\begin{array}{ccc}
1 & \sin x_{7} \tan x_{8} & \cos x_{7} \tan x_{8} \\
0 & \cos x_{7} & -\sin x_{7} \\
0 & \frac{\sin x_{7}}{\cos x_{8}} & \frac{\cos x_{7}}{\cos x_{8}}
\end{array}\right)\left(\begin{array}{l}
x_{4} \\
x_{5} \\
x_{6}
\end{array}\right) \text {, } \\
& \left(\begin{array}{c}
\dot{x}_{10} \\
\dot{x}_{11} \\
\dot{x}_{12}
\end{array}\right)=\mathbf{R}_{B E}^{-1}\left(x_{7}, x_{8}, x_{9}\right)\left(\begin{array}{c}
x_{1} \\
x_{2} \\
x_{3}
\end{array}\right)
\end{aligned}
$$

In (2) the forces $\mathbf{F}$ and the moments $\mathbf{T}$ have the following expressions:

$$
\begin{aligned}
& \mathbf{F}=\mathbf{F}_{A}+\mathbf{F}_{E}+\mathbf{F}_{G}, \\
& \mathbf{T}=\mathbf{T}_{A}+\mathbf{T}_{E},
\end{aligned}
$$

where $\mathbf{F}_{A}$ (resp. $\mathbf{T}_{A}$ ) is the aerodynamic force (resp. moment) vector, $\mathbf{F}_{E}$ (resp. $\mathbf{T}_{E}$ ) is the engine force (resp. moment) vector and $\mathbf{F}_{G}$ is the gravity force. As for the aerodynamic forces and moments, we have

$$
\begin{aligned}
\mathbf{F}_{A} & =\frac{1}{2} \rho V^{2} S \mathbf{C}_{F}(\mathbf{x}, \mathbf{u}, \mathbf{w}), \\
\mathbf{T}_{A} & =\frac{1}{2} \rho V^{2} S \mathbf{C}_{T}(\mathbf{x}, \mathbf{u}, \mathbf{w}) l_{c},
\end{aligned}
$$

where the force and moment coefficients $\mathbf{C}_{F}(\mathbf{x}, \mathbf{u}, \mathbf{w})$ and $\mathbf{C}_{T}(\mathbf{x}, \mathbf{u}, \mathbf{w})$ are nonlinear functions of the system state and of the surface deflections. These coefficients are tuned on the basis of approximate semi-heuristic methods, computational fluid dynamics, or an experimental wind tunnel or flight database.

For what concerns the measured outputs, we assume that we have at our disposal inertial sensors, such as accelerometers and rate gyros, and air data sensors. Therefore, the output vector is the following:

$$
\mathbf{y}=\left(\begin{array}{lllllllll}
V & \alpha & \beta & p & q & r & a_{x} & a_{y} & a_{z}
\end{array}\right)^{T} .
$$

Putting together (2) and (5), the aircraft equations can be rewritten as

$$
\begin{aligned}
& \dot{\mathbf{x}}=\mathbf{f}(\mathbf{x}, \mathbf{u}, \mathbf{w}), \\
& \mathbf{y}=\mathbf{h}(\mathbf{x}, \mathbf{u}, \mathbf{w}) .
\end{aligned}
$$

As is usually done, the output observer will be designed on the basis of a model obtained by linearizing the nonlinear model (6). Given a trim operation point $\overline{\mathbf{x}}$ with a corresponding input $\overline{\mathbf{u}}$, the linearized model appears in the form

$$
\begin{aligned}
& \dot{\delta \mathbf{x}}=\mathbf{A} \delta \mathbf{x}+\mathbf{B} \delta \mathbf{u} \\
& \delta \mathbf{y}=\mathbf{C} \delta \mathbf{x}+\mathbf{D} \delta \mathbf{u}
\end{aligned}
$$

where the $\mathbf{A}, \mathbf{B}, \mathbf{C}$ and $\mathbf{D}$ matrices are given by

$$
\begin{aligned}
& \mathbf{A}=\left.\frac{\partial \mathbf{f}}{\partial \mathbf{x}}\right|_{\substack{\mathbf{x}=\overline{\mathbf{x}} \\
\mathbf{u}=\mathbf{\overline { \mathbf { u } }}}}, \quad \mathbf{B}=\left.\frac{\partial \mathbf{f}}{\partial \mathbf{u}}\right|_{\substack{\mathbf{x}=\overline{\mathbf{x}} \\
\mathbf{u}=\overline{\mathbf{u}}}}, \\
& \mathbf{C}=\left.\frac{\partial \mathbf{h}}{\partial \mathbf{x}}\right|_{\substack{\mathbf{x}=\overline{\mathbf{x}} \\
\mathbf{u}=\mathbf{\overline { \mathbf { u } }}}}, \quad \mathbf{D}=\left.\frac{\partial \mathbf{h}}{\partial \mathbf{u}}\right|_{\substack{\mathbf{x}=\overline{\mathbf{x}} \\
\mathbf{u}=\overline{\mathbf{u}}}},
\end{aligned}
$$

and $\delta \mathbf{x}, \delta \mathbf{u}$ and $\delta \mathbf{y}$ indicate the variations in the states, inputs and outputs, respectively, around the trim operation point. The model (7) can be equivalently expressed in terms of a transfer matrix in the form

$$
\delta \mathbf{Y}(s)=\mathbf{G}(s) \delta \mathbf{U}(s)
$$

with $\mathbf{G}(s)=\mathbf{C}(s \mathbf{I}-\mathbf{A})^{-1} \mathbf{B}+\mathbf{D}$.

\section{Observer scheme}

In this section we will describe the observer we designed for SFDI. The driving criteria for our design were the following: 
- the development of a scheme which makes use of only one observer, so that possible implementation would be much easier;

- the scheme should allow us not only to detect the fault, but also to isolate it.

The scheme we propose is shown in Fig. 2] Starting

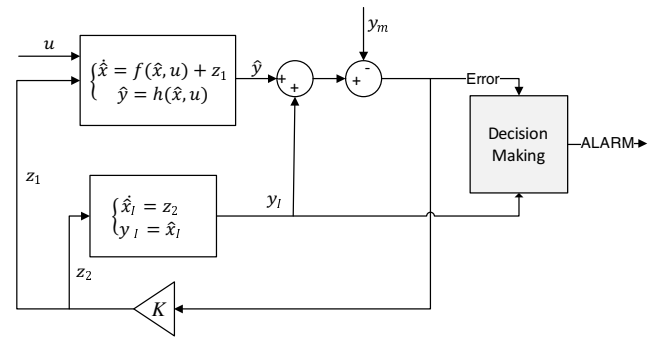

Fig. 2. SFDI scheme: in the observer, the plant is augmented with a bank of integrators, one on each variable subject to a fault. The outputs $\mathbf{y}_{\mathbf{I}}$ of these integrators are used as residuals. The observer gain $\mathbf{K}$ is constant.

from the system equations (6), we necessarily neglect the unknown disturbances $\mathbf{w}$. Hence, denoting by $\mathbf{y}_{\mathbf{m}}$ the measured outputs, the estimates $\hat{\mathbf{x}}$ of the state $\mathbf{x}$ and $\hat{\mathbf{y}}$ of the output $\mathbf{y}_{\mathbf{m}}$ would at this stage be given by

$$
\begin{aligned}
\dot{\hat{\mathbf{x}}} & =\mathbf{f}(\hat{\mathbf{x}}, \mathbf{u})+\mathbf{z}_{1}, \\
\hat{\mathbf{y}} & =\mathbf{h}(\hat{\mathbf{x}}, \mathbf{u}), \\
\mathbf{z}_{1} & =\mathbf{K}_{1}\left(\hat{\mathbf{y}}-\mathbf{y}_{m}\right) .
\end{aligned}
$$

Equations (8) describe a standard state observer, where the gain $\mathbf{K}_{1}$ can be evaluated in different possible ways, as discussed in Introduction. When using the observer (8), the residual used to detect a fault is the estimation error

$$
\mathbf{e}:=\hat{\mathbf{y}}-\mathbf{y}_{m}
$$

In this paper we propose to modify the observer equations (8) adding as many integrators to the state as the number of outputs $y$. In this way the observer equations are modified as follows:

$$
\begin{aligned}
\dot{\hat{\mathbf{x}}} & =\mathbf{f}(\hat{\mathbf{x}}, \mathbf{u})+\mathbf{z}_{1}, \\
\dot{\mathbf{x}}_{I} & =\mathbf{z}_{2}, \\
\hat{\mathbf{y}} & =\mathbf{h}(\hat{\mathbf{x}}, \mathbf{u}), \\
\mathbf{y}_{I} & =\mathbf{x}_{I}, \\
\left(\begin{array}{l}
\mathbf{z}_{1} \\
\mathbf{z}_{2}
\end{array}\right) & =\left(\begin{array}{l}
\mathbf{K}_{1} \\
\mathbf{K}_{2}
\end{array}\right)\left(\hat{\mathbf{y}}+\mathbf{y}_{I}-\mathbf{y}_{m}\right) .
\end{aligned}
$$

Hence, with respect to the standard observer-based designs for FDI, the novelty we propose is the adoption of a bank of integrators, one on each variable subject to a fault. In our scheme, as a residual we use the outputs $\mathbf{y}_{I}$ of the integrators.
The filter gain $\left(\mathbf{K}_{1}^{T} \mathbf{K}_{2}^{T}\right)^{T}$ was designed as a Kalman filter on the basis of a linearized model of the plant in the form (7). Hence, the filter gain is fixed (i.e., time invariant), whereas in the observer equations 9a and $9 \mathrm{c}$ we update the system dynamics according to the true nominal nonlinear behavior, neglecting the external disturbance $\mathbf{w}$. Indeed,

The adopted scheme meets our design criteria.

- a single observer is needed for all the possible faults, and this observer is driven by all the system outputs;

- in the ideal case when the model is known without uncertainties, and no disturbance $\mathbf{w}$ is present, the integrator outputs $\mathbf{y}_{I}$ are zero unless a sensor is faulty. Moreover, since we add a bank of integrators, when there is a fault on the $i$-th sensor, only the $i$-th integrator starts charging. In this way also the fault isolation can be achieved. This property is proven in Appendix, under some simplifying assumptions.

\section{Simulation results}

The FDI scheme described in Section 3 was massively tested in simulation in a significant number of scenarios. These scenarios were classified on the basis of

- the flight condition in the flight envelope: this was specified in terms of the operation point around which the model (7) was derived and the simulation started;

- the manoeuvre to be carried out;

- external disturbances and measurement noise;

- model uncertainties;

- the type of faults considered.

As far as the flight conditions are concerned, we considered several situations of straight wing levelled flight, at different altitudes and velocities. The manoeuvres that were analysed are shown in Table 2 As an external disturbance, we considered a continuous moderate Von Karman turbulence and a discrete gust with amplitude $\mathbf{v}_{B \text { wind }}=\left(\begin{array}{lll}3.5 & 3.5 & 3.0\end{array}\right) \mathrm{m} / \mathrm{s}$ and length $\left(\begin{array}{lll}120 & 120 & 80\end{array}\right) \mathrm{m}$. To model system uncertainties, in the simulation we modified the stability and control derivatives in the model equations (6); finally, the types of considered faults are shown in Table 3

In the sequel, we show the results of two different simulations; both were carried out in the most challenging case, i.e., in the presence of external disturbances and model uncertainties. Moreover, the measurements were also corrupted by noise. 
Table 2. List of manoeuvres considered in the simulations

\begin{tabular}{|c||c|}
\hline M01 & Elevator doublet: $5 \operatorname{deg}$ for $2 \mathrm{~s},-5 \operatorname{deg}$ for $2 \mathrm{~s}$ \\
\hline M02 & Aileron doublet: $5 \operatorname{deg}$ for $2 \mathrm{~s},-5 \operatorname{deg}$ for $2 \mathrm{~s}$ \\
\hline M03 & Rudder doublet: 2 deg for $2 \mathrm{~s},-2 \operatorname{deg}$ for $2 \mathrm{~s}$ \\
\hline M04 & Throttle variation of 0.1 \\
\hline
\end{tabular}

Table 3. List of the faults considered.

\begin{tabular}{|c||c|c|}
\hline & Bias & Drift \\
\hline Accelerometers & $\times$ & $\times$ \\
\hline Gyro rates & $\times$ & $\times$ \\
\hline Air data sensors & $\times$ & $\times$ \\
\hline
\end{tabular}

In Fig. 3 we show the residuals (i.e., the outputs of the integrators) in the case of a fault on $p$ consisting of a slow drift of $2 \mathrm{deg} / \mathrm{s}^{2}$ summed at $t=5 \mathrm{~s}$ at the simulation output.

In Fig. 4 we show the residuals in the case of a fault on $a_{z}$ consisting of a bias simulated as a step of $1 \mathrm{~m} / \mathrm{s}^{2}$ occurring at $t=5 \mathrm{~s}$. In both the cases, when the fault occurs, the integrator linked to the faulty variable starts charging significantly more than the integrators linked to the other variables. These behaviours seem promising for FDI, as will be shown in the next section, where we make use of flight recorded data rather than simulated data.

4.1. Comparison with a standard FDI scheme. For the sake of comparison, we implemented the standard FDI scheme based on a Kalman filter, described by Eqns. (8) and shown in Fig. 5. In this comparison, we considered the case of a fault on $p$ simulated as a step signal of $5 \mathrm{deg} / \mathrm{s}$ occurring at $t=10 \mathrm{~s}$. Figure 6 shows the behaviour of the two observers, i.e., the standard Kalman filter (KF) and the proposed one augmented with integrators (KFI). The latter one performs much better since, as expected, it shows less coupling between the residuals of a faulted variable and the other ones.

\section{Results using experimental flight data}

In this section we test the proposed observer based SFDI scheme on some flight data. In order to make the implementation closer to that on a real aircraft, a sensor FDI technique should be able to detect and isolate faults in the presence of external disturbances, unmodelled dynamics, and neglected nonlinearities. On the other hand, an important feature is the minimization of missed and false alarms, which will be dealt with a proper threshold tuning technique based on an ad-hoc decision making system, presented in Section 5.1 .

We adopted the following procedure:
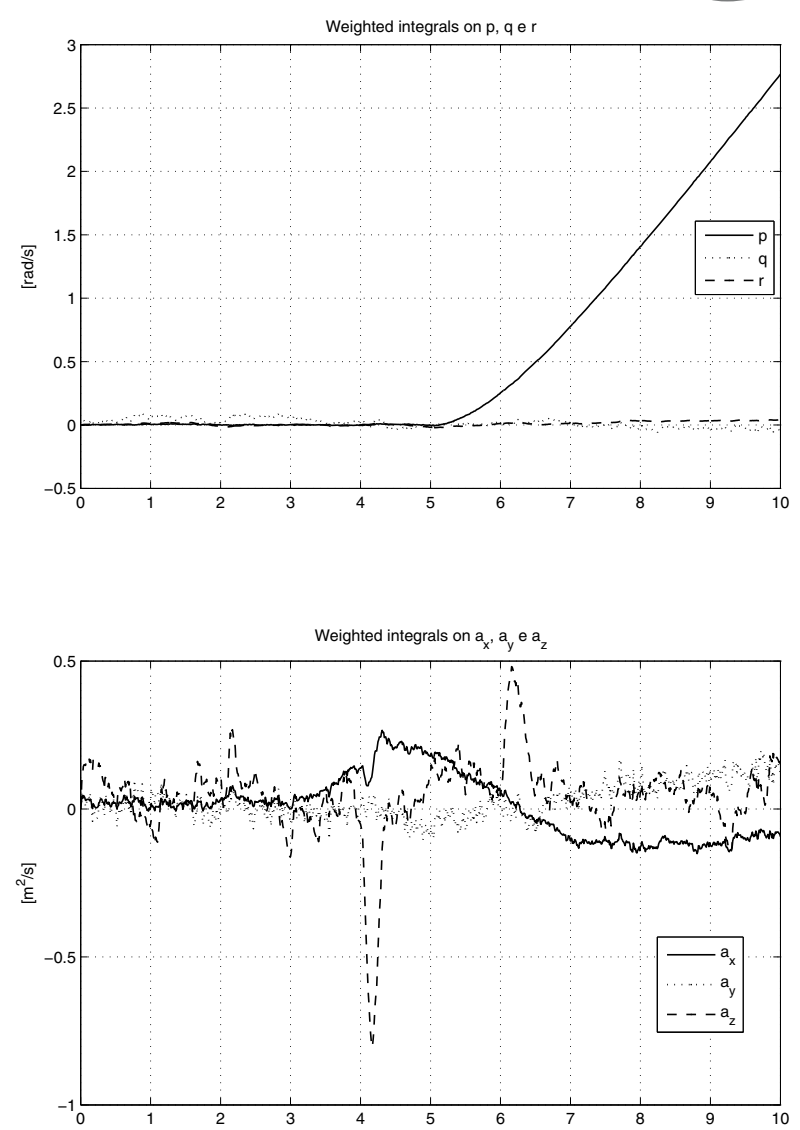

Fig. 3. Simulation in the presence of a fault on $p$ consisting of a slow drift of $2 \mathrm{deg} / \mathrm{s}^{2}$ at $t=5 \mathrm{~s}$. The two panels show the state of the observer integrators. The residual linked to $p$ is significantly larger than the others.

- Starting from the experimental data, a time instant when the angle of attack $\alpha$ and the velocity $V$ are constant was found to catch steady-state conditions.

- The nonlinear model (6) was trimmed at the time instant determined before and the linearized model (7) was derived.

- Based on this linearized model, a gain for the observer derived from the Kalman filter was calculated. This gain was kept constant in our simulations.

- A simulation using the experimental data was carried out in the presence of faults.

- The residuals, i.e., the output of the integrators, were used to detect and isolate the fault.

For fault detection, we adopted the decision making process described in the next section. 

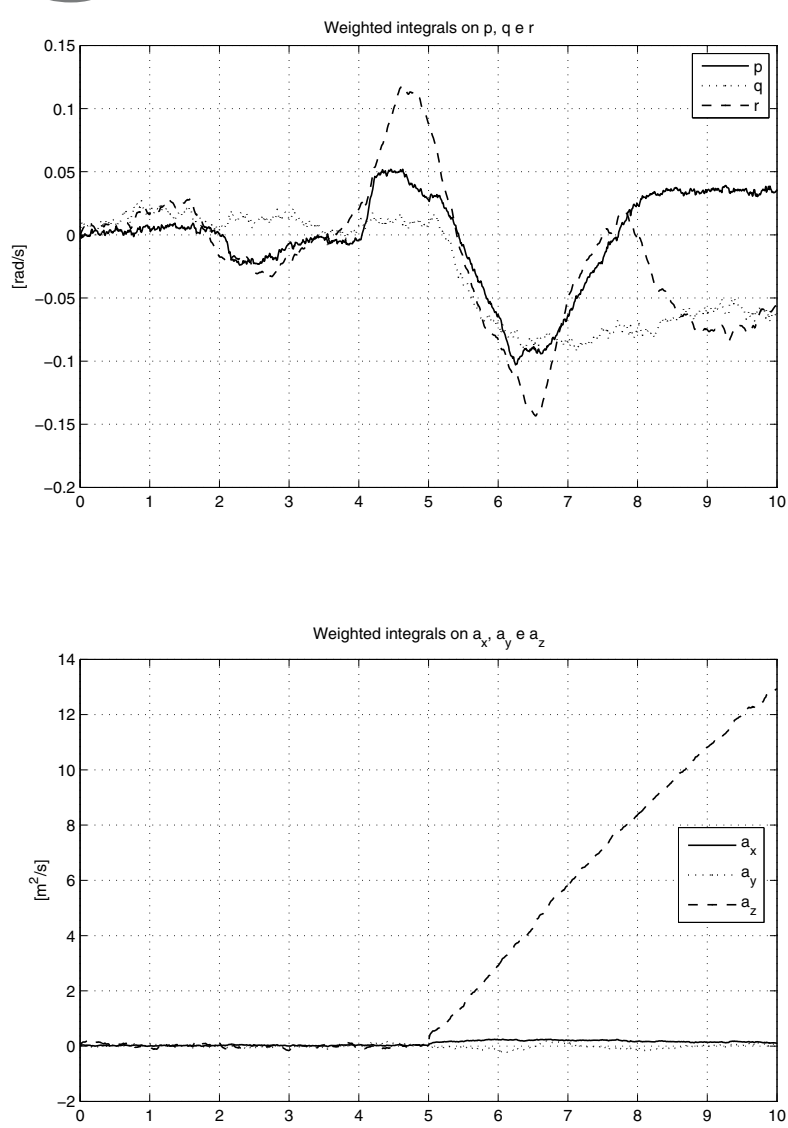

Fig. 4. Simulation in the presence of a fault on $a_{z}$ consisting of a bias simulated as a step of $1 \mathrm{~m} / \mathrm{s}^{2}$ occurring at $t=5 \mathrm{~s}$. The two panels show the state of the observer integrators. The residual linked to $a_{z}$ is significantly larger than the others.

5.1. Decision making algorithm. The role of the proposed decision-making algorithm is to decide if a fault can be detected and isolated on the basis of the residuals' time histories. Due to the stochastic nature of the process under consideration, which is subject to unpredictable disturbances and uncertainties, the problem must be approached stochastically and one has to fix an acceptable level of false and/or missed alarm probabilities. When the algorithm is based on a threshold logic, the values of thresholds can be selected on the basis of minimum false and missed alarm desired probabilities, say $P_{F A \text { min }}$ and $P_{M A \text { min }}$.

If we denote by $r_{i}(k)$ the value of the $i$-th residual, which in our case is the output of the $i$-th integrator $\mathbf{y}_{I}$ in $9 \mathrm{dd}$, at time $k$, the mathematical problem to tune the value $\gamma_{i}$ of the threshold on measurement channel $i$ is

- $\operatorname{Prob}\left(\left|\bar{r}_{i}(k)\right|>\gamma_{i}\right) \leq P_{F A \text { min }}$ for all $k$ when the $i$-th measurement is not faulty,

- $\operatorname{Prob}\left(\left|\bar{r}_{i}(k)\right| \leq \gamma_{i}\right) \leq P_{M A \min }$ for all $k>k_{f}+\Delta$,

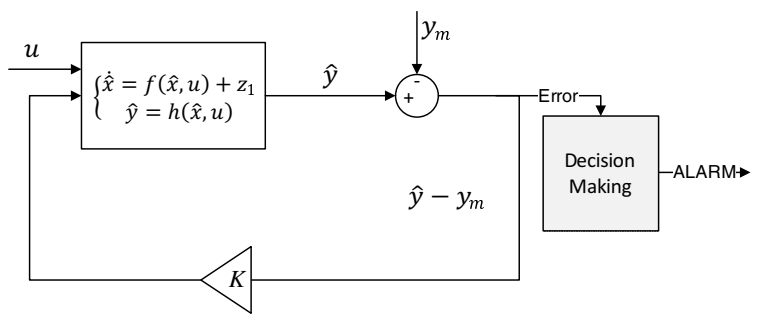

Fig. 5. Scheme of a standard observer-based FDI system.

where $k_{f}$ is the fault time and $\Delta$ is the allowed delay detection time.

By $\bar{r}_{i}(k)$ we indicate a filtered version of the signal $r_{i}(k)$, obtained by means of a moving-average algorithm.

Let us denote by $\bar{r}_{N F_{i}}(k)$ the residual value function restricted to the time instants when no faults occur and by $\bar{r}_{F_{i}}(k)$ the residual value function restricted to the time instants when faults occur.

In Fig. 7, we show an ideal case where the PDFs (probability density functions) of processes $\bar{r}_{N F_{i}}(k)$ and $\bar{r}_{F_{i}}(k)$ are Gaussians. The PDF of $\bar{r}_{F_{i}}(k)$ is replicated for two different amplitudes of the faults. Let us call them "weak" and "strong" faults. The two PDFs are well separated if we consider the strong fault but they have a non-negligible intersection in the weak fault case. Once a threshold on the residual function value is decided, also the probability of missed and false alarms (areas A and B in Fig. 7, respectively) is fixed.

In our application, we decided to achieve the probability of false alarms as low as possible. This choice is acceptable if the category of faults which run the risk to be masked by the FDI algorithm (missed alarms) can be readily rejected by the control system or by the pilot. If we want to completely avoid false alarms, $P_{F A}$ min must be fixed to as zero and the threshold tuning problem can be then translated into the following constrained linear minimization problem $\left(L_{i}=\gamma_{i}^{-1}\right)$ :

$$
\min _{L_{i}}-L_{i}
$$

subject to

$$
\left\{\begin{array}{l}
L_{i}>0 \\
L_{i}\left|\bar{r}_{i}(k)\right|<1 \forall k \quad \text { in the absence of faults. }
\end{array}\right.
$$

The above optimization problem was solved using experimental data extracted from flight recordings to build $\bar{r}_{i}(k)$. Two flights, each one lasting about $1800 \mathrm{~s}$, were used. These data were processed with a 10-point moving average filter with a time step of $0.1 \mathrm{~s}$. The fault is detected when the threshold is exceeded for at least five consecutive samples. 

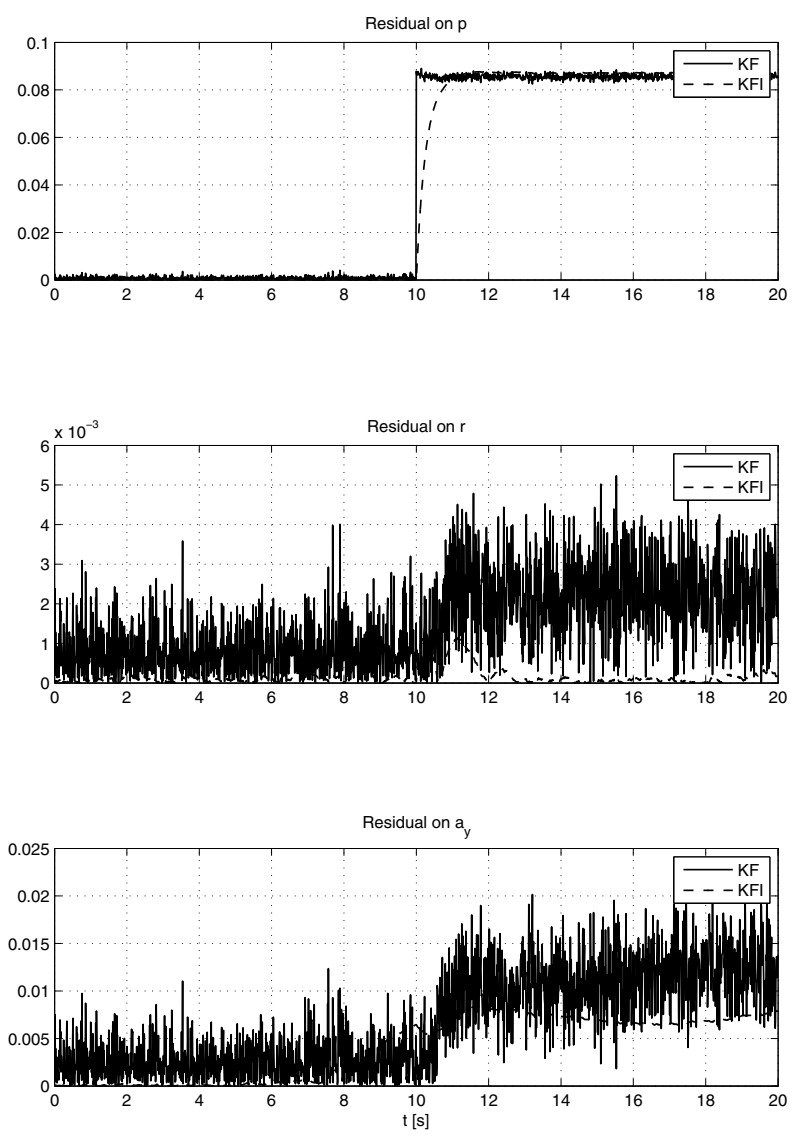

Fig. 6. Comparison between residuals of a standard Kalman filter (KF) and the proposed scheme (KFI). The three plots show the residuals in the presence of a fault on $p$, consisting of a step of amplitude of $5 \mathrm{deg} / \mathrm{s}$ occurring at $t=10 \mathrm{~s}$. The residuals for the KFI scheme show less coupling.

Figure 8 shows the experimental PDFs on the $p$ measurement channel when a bias fault is considered. The residual distributions in the case of no-fault and biases with an amplitude of 3 and $5 \mathrm{deg} / \mathrm{s}$ show that fixing a threshold around 0.06 nullifies in practice the occurrence of false alarms with a very low probability of having missed alarms for a $5 \mathrm{deg} / \mathrm{s}$ bias, whereas a $3 \mathrm{deg} / \mathrm{s} \mathrm{bias}$ can be considered a rejectable offset. Figure 9 shows three experimental residual PDFs for the following cases with respect to the $p$ measurement channel: (i) no fault, (ii) a bias fault with an amplitude of $5 \mathrm{deg} / \mathrm{s}$, (iii) a drift of $2 \mathrm{deg} / \mathrm{s}^{2}$. A threshold around 0.06, which is the output of the proposed linear optimization problem, nullifies in practice the occurrence of false alarms with a very low probability of having missed alarms for the two fault categories considered.

5.2. Discussion of the results. The observer-based SFDI scheme was tested on recorded flight data to which

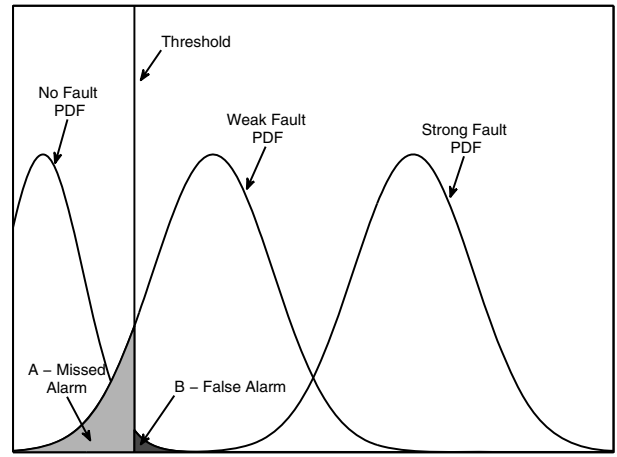

Fig. 7. Example of an ideal residual PDF in the absence of faults, and in the presence of weak and strong faults. The value of the threshold for decision making determines the probability of missed and false alarms (areas A and $\mathrm{B}$, respectively).

fault signals were artificially added. A total number of 12 faulty cases were considered for the inertial and air data sensors.

Hereafter we present some of the results, both in terms of residuals' time histories, as well as of detection and isolation of the faults, using the decision making process described in the previous section.

The residuals, i.e., the outputs of the integrators, were weighted by the coefficients $L_{i}$ solving the optimization problem (10). In this way, if the threshold, set to 1 , is exceeded, the SFDI scheme gives a fault detection alarm.

In Fig. 10 we show the weighted residuals in the case of a fault on $p$ consisting of a slow drift of $5 \mathrm{deg} / \mathrm{s}^{2}$ occurring at $630 \mathrm{~s}$.

In Fig. 11 we show the weighted residuals in the case of a fault on $a_{x}$ consisting of a bias simulated as a step of $5 \mathrm{~m} / \mathrm{s}^{2}$ occurring at $630 \mathrm{~s}$.

If we compare these results with those obtained in Section 4 , where simulated data were used, we can notice that the residuals are more coupled in this case. The main reason is that, since we are now using recorded flight data, all the unmodelled dynamics and the model uncertainty are causing the charging of the integrators.

Nevertheless, choosing the thresholds as discussed in Section 5.1, we were able to detect and isolate the faults in all the cases. Table 4 shows the time needed to detect the faults in the twelve cases that were considered for the inertial and air data sensors.

\section{Conclusions}

In this paper we presented a scheme for sensor fault detection and isolation based on analytical redundancy. The scheme consists of an observer designed using the 

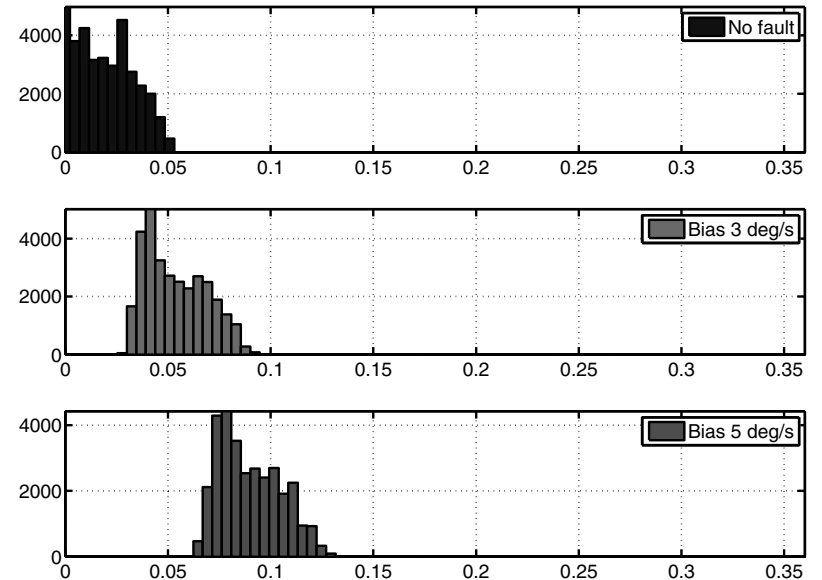

Fig. 8. Residual PDFs computed by means of experimental data in the absence of faults, and in the presence of weak and strong biases on $p$ measurements ( 3 and $5 \mathrm{deg} / \mathrm{s}^{2}$, respectively).

Table 4. Delays in the detection and isolation of the faults for the various cases considered.

\begin{tabular}{|c||c|}
\hline Fault & Detection delay [s] \\
\hline Step on $p$ of $5 \mathrm{deg} / \mathrm{s}$ & 4.5 \\
\hline Drift on $p$ of $5 \mathrm{deg} / \mathrm{s}^{2}$ & 2.8 \\
\hline Step on $q$ of $5 \mathrm{deg} / \mathrm{s}$ & 1.1 \\
\hline Drift on $q$ of $5 \mathrm{deg} / \mathrm{s}^{2}$ & 2.9 \\
\hline Step on $r$ of $5 \mathrm{deg} / \mathrm{s}$ & 1.3 \\
\hline Drift on $r$ of $5 \mathrm{deg} / \mathrm{s}^{2}$ & 1.7 \\
\hline Step on $a_{x}$ of $5 \mathrm{~m} / \mathrm{s}^{2}$ & 6.5 \\
\hline Drift on $a_{x}$ of $2 \mathrm{~m} / \mathrm{s}^{3}$ & 6.2 \\
\hline Step on $a_{y}$ of $5 \mathrm{~m} / \mathrm{s}^{2}$ & 6.5 \\
\hline Drift on $a_{y}$ of $2 \mathrm{~m} / \mathrm{s}^{3}$ & 6.1 \\
\hline Step on $a_{z}$ of $5 \mathrm{~m} / \mathrm{s}^{2}$ & 3.6 \\
\hline Drift on $a_{z}$ of $2 \mathrm{~m} / \mathrm{s}^{3}$ & 4.5 \\
\hline
\end{tabular}

Kalman filter approach, applied to the nominal nonlinear plant augmented with a bank of integrators, one for each sensor that is subject to faults.

One advantage of the proposed scheme is the fact that it needs only one observer, which is driven by all the system outputs. If a fault occurs on a generic sensor, the corresponding integrator starts charging; therefore, the states of the integrators are assumed as residuals for the FDI.

The proposed approach was tested both using simulated data, assuming the presence of noise, external disturbances and model uncertainties, and using recorded flight data. It was shown that it can be effectively used to detect and isolate faults on inertial sensors or on air data sensors.

Future extensions of the present work will cover the

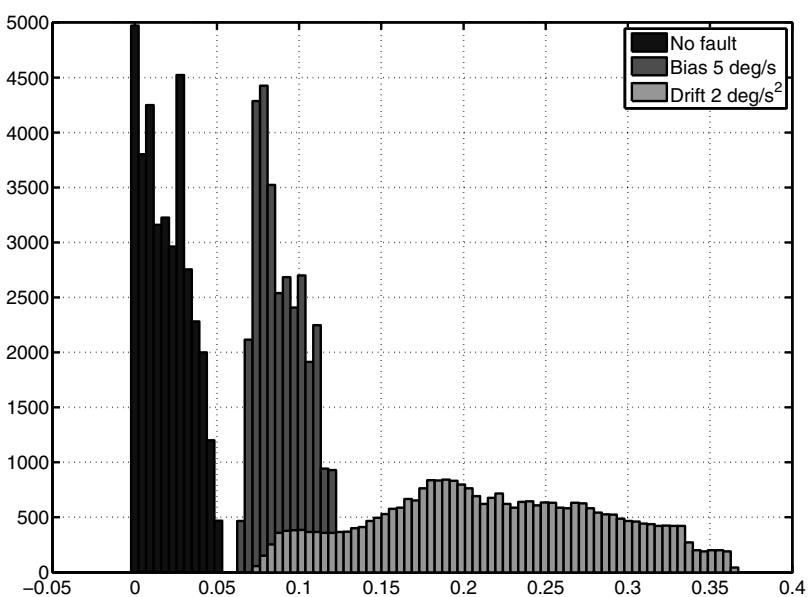

Fig. 9. Residual PDFs computed by means of experimental data in the absence of faults, and in the presence of biases and drifts on $p$ measurements (amplitudes of $5 \mathrm{deg} / \mathrm{s}$ and 2 $\mathrm{deg} / \mathrm{s}^{2}$, respectively).

following issues: the behaviour of the observer scheme in the presence of uncertainties and optimization of the filter taking into account the presence of wind.

\section{References}

Basseville, M. and Nikiforov, I.V. (1993). Detection of Abrupt Changes: Theory and Application, Prentice Hall, Englewood Cliffs, NJ.

Chen, J. and Patton, R.J. (1999). Robust Model-Based Fault Diagnosis for Dynamic Systems, Kluwer Academic Publishers, Norwell, MA.

Clark, R.N. (1978). A simplified instrument failure detection scheme, IEEE Transactions on Aerospace and Electronic Systems 14(4): 558-563.

Clark, R.N., Fosth, D.C. and Walton, V.M. (1975). Detecting instrument malfunctions in control systems, IEEE Transactions on Aerospace and Electronic Systems 11(4): 465-473.

Gertler, J. (1997). Fault detection and isolation using parity relations, Control Engineering Practice 5(5): 653-661.

Gertler, J. (1998). Fault Detection and Diagnosis in Engineering Systems, Marcel Dekker, New York, NY.

Gustafsson, F. (2000). Adaptive Filtering and Change Detection, Wiley, New York, NY.

Isermann, R. (1984). Process fault detection based on modeling and estimation methods: A survey, Automatica 20(4): 387-404.

Kalman, R.E. (1960). A new approach to linear filtering and prediction problems, Journal of Basic Engineering 82(1): 35-45.

Luenberger, D.G. (1971). An introduction to observers, IEEE Transactions on Automatic Control 16(6): 596-602. 

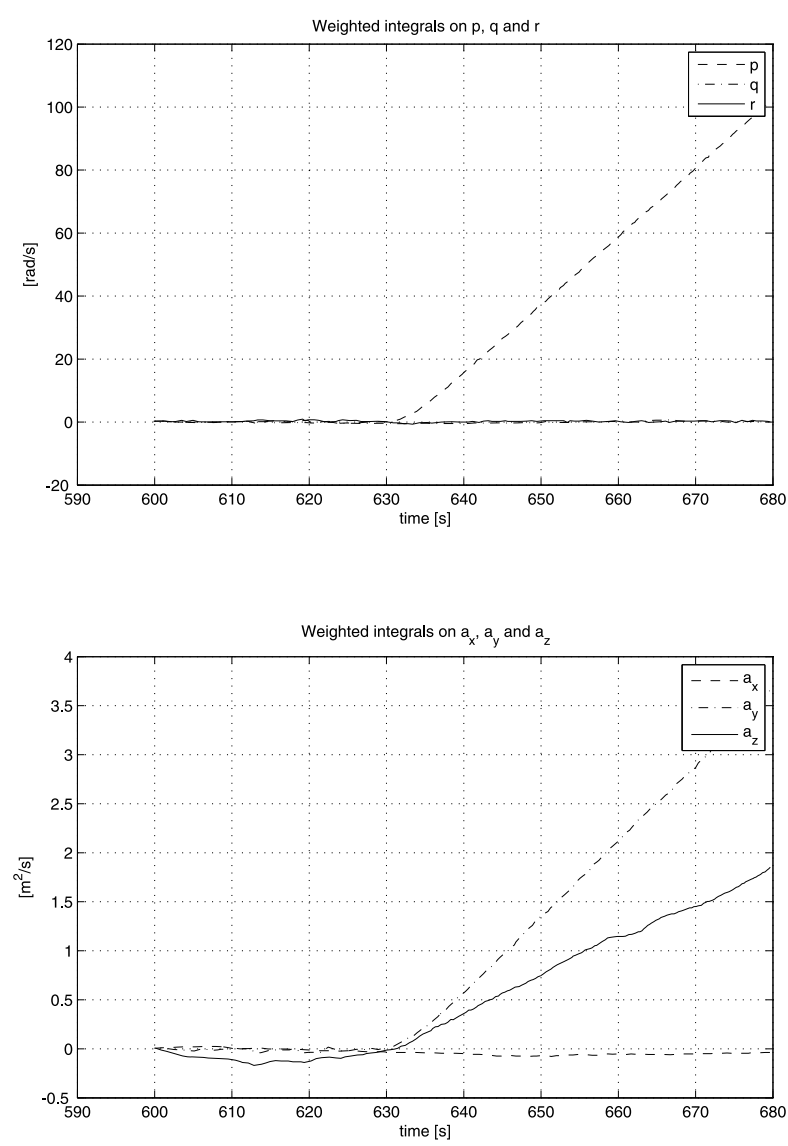

Fig. 10. Simulation using recorded flight data in the presence of a fault on $p$ consisting of a slow drift of $5 \mathrm{deg} / \mathrm{s}^{2}$ at $630 \mathrm{~s}$. The two figures show the state of the observer integrators, weighted by the coefficients $L_{i}$ solving the optimization problem $[10$. The fault is detected when the threshold, set to 1 , is exceeded.

Mattei, M., Paviglianiti, G. and Scordamaglia, V. (2005). Nonlinear observers with H-infinity performance for sensor fault detection and isolation: A linear matrix inequality design procedure, Control Engineering Practice 13(10): 1271-1281.

Mehra, R.K. and Peschon, J. (1971). An innovations approach to fault detection and diagnosis in dynamic systems, Automatica 7(5): 637-640.

Patton, R.J. and Chen, J. (1994). A review of parity space approaches to fault diagnosis for aerospace systems, Journal of Guidance, Control, and Dynamics 17(2): 278-285.

Simani, S., Fantuzzi, C. and Patton, R.J. (2003). Model-based Fault Diagnosis in Dynamic Systems Using Identification Techniques, Springer-Verlag, New York, NY.

Stevens, B.L. and Lewis, F.L. (2003). Aircraft Control and Simulation, 2nd Edn., John Wiley \& Sons, Inc., Hoboken, NJ.

Watanabe, K. and Himmelblau, D.M. (1982). Instrument fault detection in system with uncertainties, International Journal of Systems Science 13(2): 137-158.

Wünnenberg, J. and Frank, P.M. (1987). Sensor fault detection via robust observers, in S. Tzafestas, M. Singh and G.
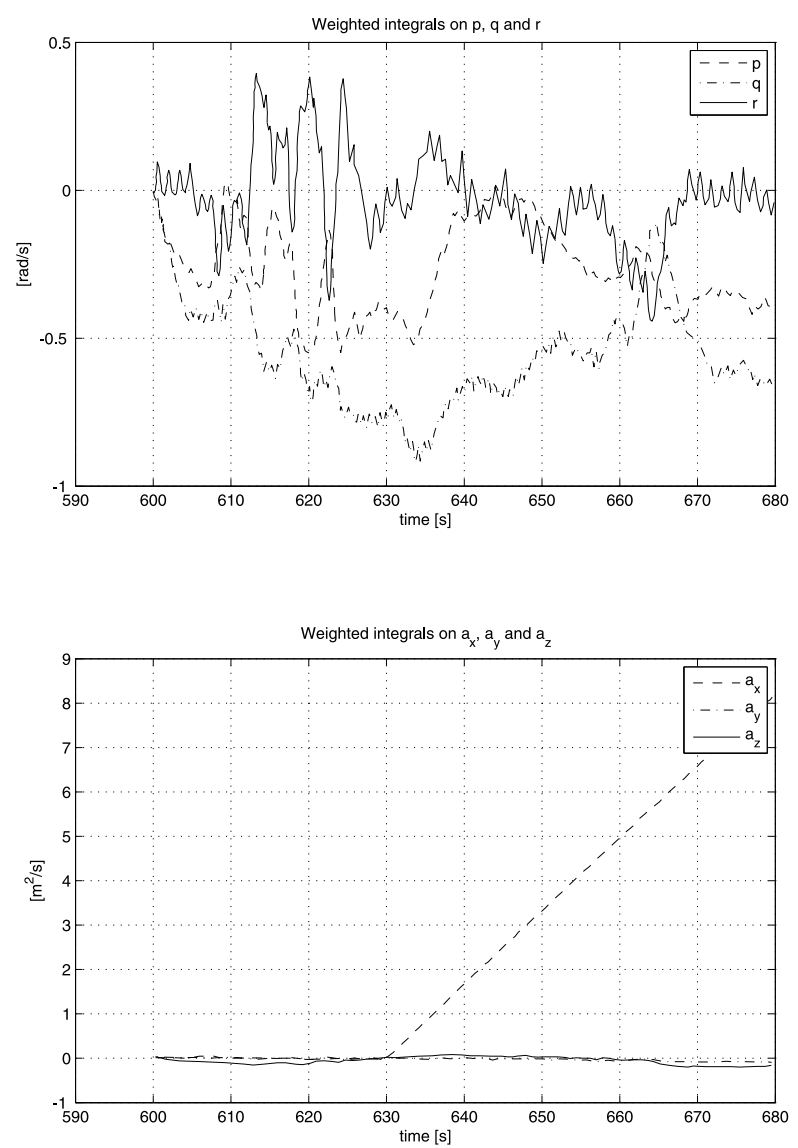

Fig. 11. Simulation using recorded flight data in the presence of a fault on $a_{x}$ consisting of a bias simulated as step of $5 \mathrm{~m} / \mathrm{s}^{2}$ occurring at $630 \mathrm{~s}$. The two figures show the state of the observer integrators, weighted by the coefficients $L_{i}$ solving the optimization problem (10). The fault is detected when the threshold, set to 1 , is exceeded.

Schmidt (Eds.), System Fault Diagnostics, Reliability \& Related Knowledge-based Approaches, Vol. 1, D. Reidel Publishing Company, Dordrecht, pp. 147-160.

Yeh, Y.C. (1996). Triple-triple redundant 777 primary flight computer, Proceedings of the Aerospace Applications Conference, Aspen, CO, USA, pp. 293-307.

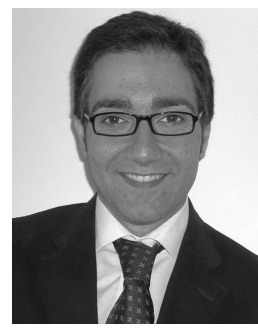

Marco Ariola was born in Naples, Italy, in 1971. $\mathrm{He}$ received the laurea degree in electronic engineering and the research doctorate degree in electronic engineering and computer science from the University of Naples Federico II, in 1995 and 2000, respectively. From 1996 to 2005 he was with the Department of Computer and Systems Engineering of the University of Naples Federico II. Currently, he is a full professor of automatic control at the University of Naples Parthenope in the Engineering Department, where he is the coordinator of the Ph.D. programme in information engineering. His research interests include statistical control, robust control, control of nuclear fusion devices, control of aerospace systems. He has published more than 160 journal papers, conference papers, and articles in books and encyclopaedias. He 
has served as an associate editor of the IEEE Control System Society Conference Editorial Board since 2006. He has been an IEEE senior member since 2006.

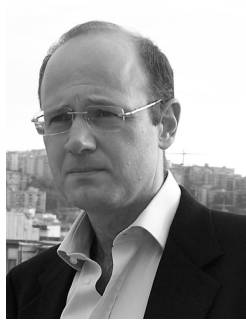

Massimiliano Mattei received the Laurea degree in aeronautical engineering and the Ph.D. degree in electronic engineering from the University of Napoli Federico II (Italy), in 1993 and 1997, respectively. He is a full professor of flight mechanics and control at the Second University of Napoli, where he is also the vice-rector for research, informatics and evaluation, and the scientific coordinator of the Laboratory of Flight Dynamics and Control. He has been a scientific consultant for the Italian Aerospace Research Center and for the ENEA/CREATE association. He has published more than 150 scientific papers. He has served as an associate editor of IEEE Transactions on Control System Technology. He is an AIAA and an IEEE senior member. His research interests are in the area of control theory and applications, flight control, unmanned aerial vehicles, mathematical modeling of complex systems, thermonuclear controlled fusion, and fault detection and isolation.

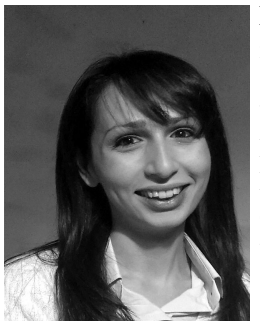

Immacolata Notaro was born in Naples, Italy, in 1985. She received the Master's degree cum laude in aeronautical engineering at the Second University of Naples in 2013. Since 2014, she has been a Ph.D. student at the same faculty and her research topics cover fault detection and redundancy, as well as predictive control, applied to autonomous navigation of unmanned aircraft and swarms.

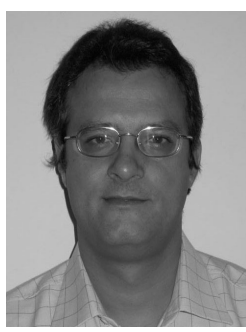

Federico Corraro graduated cum laude in electronics engineering in 1992 with a Ph.D. in computer engineering. As a research engineer in the Flight System Department (currently the Guidance Navigation \& Control Department) at CIRA since 1994, he has been involved in several national and international projects mainly concerning flight mechanics, sense and avoid, and advanced guidance, navigation and control of both manned and unmanned aerospace vehicles. An author of over 100 scientific papers on the subjects, he was also a contract professor of control system technologies at the Department of $\mathrm{Au}-$ tomation Engineering of the University of Naples Federico II (from 2001 to 2004). Currently, he is the head of the Information Technologies Unit at CIRA.

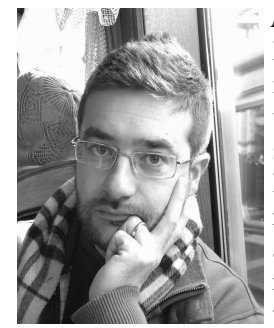

Adolfo Sollazzo was born in Capua, Italy, 1975. He received a degree in electronic engineering in 2001 and a Ph.D. in electrical energy conversion in 2005, both from the Second University of Naples. He joined the Italian Aerospace Research Centre (CIRA) in 2003, where he currently works as a senior researcher at the Guidance, Navigation and Control Lab. His field of research presently covers several aspects of autonomous flight for manned and unmanned space and air vehicles, particular fault-tolerance and multivariable control.

\section{Appendix}

In this appendix we show that, considering a linearized model of the aircraft, the SFDI scheme proposed in Section 3 guarantees detection and isolation.

Let us consider the scheme shown in Fig. A1, which is described by the following equations:

$$
\begin{aligned}
\delta \hat{\mathbf{y}} & =\mathbf{G}(s) \delta \mathbf{u}+\mathbf{H}(s) \mathbf{z}_{1}, \\
\delta \mathbf{y}_{m} & =\mathbf{G}(s) \delta \mathbf{u}, \\
\mathbf{y}_{I} & =\frac{\mathbf{I}}{s} \mathbf{z}_{2}, \\
\left(\begin{array}{l}
\mathbf{z}_{1} \\
\mathbf{z}_{2}
\end{array}\right) & =\left(\begin{array}{l}
\mathbf{K}_{1} \\
\mathbf{K}_{2}
\end{array}\right)\left(\delta \hat{\mathbf{y}}+\mathbf{y}_{I}-\delta \mathbf{y}_{m}-\mathbf{d}\right) .
\end{aligned}
$$

We are interested in calculating the DC gain of the

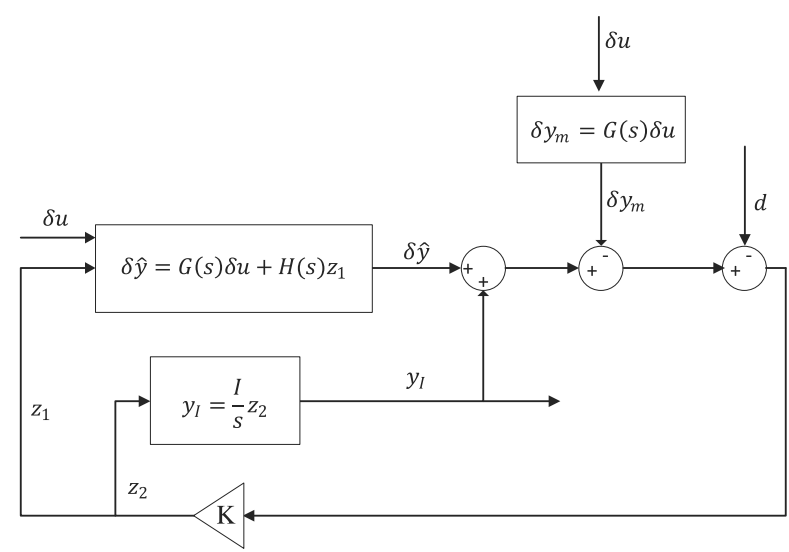

Fig. A1. Linearized version of the SFDI scheme.

transfer matrix linking $\mathbf{d}$ to $\mathbf{y}_{I}$. Solving Eqn. A1d for $z_{2}$, we obtain

$$
\begin{aligned}
\mathbf{z}_{2} & =\mathbf{K}_{2}\left[\mathbf{H}(s)\left(\mathbf{I}-\mathbf{K}_{1} \mathbf{H}(s)\right)^{-1} \mathbf{K}_{1}+\mathbf{I}\right]\left(\mathbf{y}_{I}-\mathbf{d}\right) \\
& =\mathbf{K}_{2}\left[\mathbf{H}(s) \mathbf{K}_{1}\left(\mathbf{I}-\mathbf{H}(s) \mathbf{K}_{1}\right)^{-1}+\mathbf{I}\right]\left(\mathbf{y}_{I}-\mathbf{d}\right) \\
& =\mathbf{K}_{2}\left(\mathbf{I}-\mathbf{H}(s) \mathbf{K}_{1}\right)^{-1}\left(\mathbf{y}_{I}-\mathbf{d}\right) .
\end{aligned}
$$

Now, using (A1c), Eqn. (A2) becomes

$$
s \mathbf{y}_{I}=\mathbf{K}_{2}\left(\mathbf{I}-\mathbf{H}(s) \mathbf{K}_{1}\right)^{-1}\left(\mathbf{y}_{I}-\mathbf{d}\right) .
$$

After some manipulations, assuming that $\mathbf{K}_{\mathbf{2}}$ is invertible, Eqn. A3 can be solved for $\mathbf{y}_{I}$, which yields

$$
\mathbf{y}_{I}=\left(\mathbf{I}-s\left(\mathbf{I}-\mathbf{H}(s) \mathbf{K}_{1}\right) \mathbf{K}_{2}^{-1}\right)^{-1} \mathbf{d} .
$$

Hence, assuming that the system is asymptotically stable and letting $s=0$, we have that at steady state the DC gain is equal to the identity matrix $\mathbf{I}$. Therefore, in the case of faults modelled as step variations, isolation of the fault at steady state is guaranteed. 